\title{
Impact of different polyimide-based substrates on the soft magnetic properties of NiFe thin films
} Johannes Rittinger ${ }^{*}$, Piriya Taptimthong ${ }^{1}$, Lisa Jogschies ${ }^{1}$, Marc C. Wurz ${ }^{1}$ and Lutz Rissing ${ }^{1}$ ${ }^{1}$ Institut für Mikroproduktionstechnik, Leibniz Universität Hannover, An der Universität 2, 30823 Garbsen, Germany

\begin{abstract}
We investigated the impact of polymer substrates on the magnetic properties of soft magnetic thin films. Experiments were carried out to evaluate the performance of AMR (anisotropic magnetoresistive) sensors deposited on polymeric substrates and to give indications for the design of future sensors on flexible substrates. Sputtered permalloy (NiFe 81/19) was used as a soft magnetic thin film layer. As substrate materials, liquid polyimide precursors and DuPont Kapton ${ }^{\circledR}$ HN foil were examined. Surface roughness was determined for each substrate material. The dynamic of soft magnetic behavior of the permalloy thin films was observed in a homogenous alternating magnetic field. Resulting R-Hcurves were evaluated in regard to the magnitude of the magnetoresistive effect ( $\Delta \mathrm{R} / \mathrm{R} 0$-ratio), as well as the resulting magnetic anisotropy of the tested samples. B-H-curves were obtained by means of a vibrating sample magnetometer (VSM).
\end{abstract}

Keywords: Magnetoresistive devices, Flexible substrates, Polyimides, Permalloy

\section{INTRODUCTION}

In order to meet the increasing demands towards precision and efficiency of machine tools in modern production technology, a novel approach is pursued within the Collaborative Research Center (CRC) 653 that is funded by the German Research Foundation (DFG). This novel approach is based on the attempt to meet the increasing demands by collecting information during the lifetime of a system and storing the same within the observed component. Thus, components like a machine tool or a milled part become genetically intelligent ("gentelligent" [1]). In any case, as much information as possible has to be gathered to obtain a comprehensive image of the observed component. Thus, for the realization of such a "gentelligent" unit or system, sensors with high integrability are required to provide the components with senses. Various types of micro sensors, namely strain gauges, eddy current sensors and anisotropic magnetoresistive (AMR) sensors, have been successfully implemented by applying a modular thin film fabrication technology on flexible polymer foils [2].

Nevertheless, it has to be considered that various factors such as homogeneity, morphology and surface roughness of the substrate material, to name a few, do have an impact on the properties of deposited thin films, specifically in the case of soft magnetic material [3, 4]. In literature, phenomena connected to interactions between a substrate and a ferromagnetic thin film have been investigated intensively, the striking example being exchange anisotropy [5]. This effect is observed when a ferromagnetic layer is deposited on an antiferromagnetic layer forming a ferromagnetic-antiferromagnetic interface. The resulting interactions manifest themselves as an exchange bias that can be determined experimentally as a shift of the hysteresis of the ferromagnetic layer along the field axis [6, 7]. But the interaction of a ferromagnetic thin film with its substrate is not limited to ferromagnetic-antiferromagnetic interfaces. Surface anisotropy, which was first described by Néel [8], attributes the deviant behavior of thin films from bulk samples to the reduced symmetry of the atomic environment of surface atoms and applies for magnetic thin films on any substrate.

In the design of thin film magnetoresistors, it is often assumed that the sensing element is in mono-domain state. However, the magnetic properties of the real magnetic thin film deviate from those predicted by theoretical models, such as the Stoner-Wohlfarth model. These deviations appear as the thin film is normally in the multi domain state, which causes complex domain changes during magnetization. As a result, the anisotropy of a real thin film disperses [9]. It has to be expected that this dispersion of anisotropy correlates with the homogeneity of the used substrate.

Smart Sensors, Actuators, and MEMS VII; and Cyber Physical Systems, edited by José L. Sánchez-Rojas,

Riccardo Brama, et. al., Proc. of SPIE Vol. 9517, 95171R · (C) 2015 SPIE

CCC code: $0277-786 \mathrm{X} / 15 / \$ 18 \cdot$ doi: $10.1117 / 12.2178765$ 
Besides "pure" surface related effects, another factor which has an impact on the magnetization behavior of a magnetic thin film has to be considered when using polymeric, flexible substrates. Compared to a rigid substrate, larger discrepancies between the physical properties (e.g. coefficient of thermal expansion or Young's modulus) of a polymeric substrate and a metallic thin film occur. As a result the appearance of elevated stress in the thin film is very likely. This additional stress influences the domain structure of the thin film on the microscopic scale [10] as well as the macroscopic magnetic behavior of the thin film device. Interactions of magnetic materials with external and internal stresses are attributed to magneto-elastic coupling, a topic that has been covered by numerous studies, e.g. [11].

Various groups have proven the viability of magnetic devices based on metallic thin films on flexible polymeric substrates in the past. A striking example being [12], where the impact of spacer and barrier layers on the soft magnetic performance of sputtered permalloy multilayers on a polyester substrate were investigated and thereupon magnetic properties optimized. While [12] meant to use these multilayers either as identification markers or EMI shields, [13] intended to use rather thick (in the range of several $100 \mathrm{~nm}$ ) permalloy multilayer films on cyclo olefin copolymer (COC) substrates as pressure sensors depending on the giant magneto impedance effect. Although the magnetic properties of the permalloy films were significantly affected by the used substrate, a high sensitivity of the sensors could still be achieved. The cited publications thus stand exemplary for the intensive work on soft magnetic multilayer thin films on flexible substrates.

In contrast to the above-mentioned research, the objective of this study was to investigate the impact of polymeric substrates on the soft magnetic properties of sputter deposited single and thin permalloy films. Experiments were carried out in order to evaluate the feasibility of precise AMR sensors based on such polymeric substrates. As substrate, different polyimide-based materials were examined. These substrates comprise polyimide polymerized from various spin-on precursors as well as Kapton ${ }^{\mathbb{R}} \mathrm{HN}$ foil. Polyimide-based materials were chosen as substrate material due to their superior mechanical, chemical and thermal properties [14] that are a prerequisite for the future use of such sensors in harsh industrial environments. Sputtered permalloy (NiFe 81/19), a standard material for AMR sensor applications, was used as soft magnetic thin film layer. Nominal thickness of the thin film was $100 \mathrm{~nm}$.

\section{EXPERIMENTAL}

\section{A. Sample Preparation}

A first group of specimens was fabricated on a silicon wafer with a thermal oxide top layer and served as a reference. Further specimens were prepared on four polyimide-based materials, three different liquid polyimide precursors on the one hand and DuPont Kapton ${ }^{\circledR} \mathrm{HN}$ foil on the other hand.

The precursors were spin coated onto a silicon wafer and polymerized through heat treatment at $350{ }^{\circ} \mathrm{C}$, resulting in a $6 \mu \mathrm{m}$ thick polyimide base layer. $50 \mu \mathrm{m}$ thick Kapton ${ }^{\circledR} \mathrm{HN}$ foil backed with a silicone based adhesive film was applied onto a silicon wafer for thin film processing using a commercially available laminator (Bungard Elektronik RLM 419p).

Single sensor structures featuring dimensions depicted in Fig.1 a) were chosen as exemplary magnetoresistors for the experimental determination of $R$ - $H$-curves. The represented geometry resembles the design of commercial magnetoresistors and was chosen for the single sensor elements in order to ensure strong shape anisotropy with distinct predominant directions. 


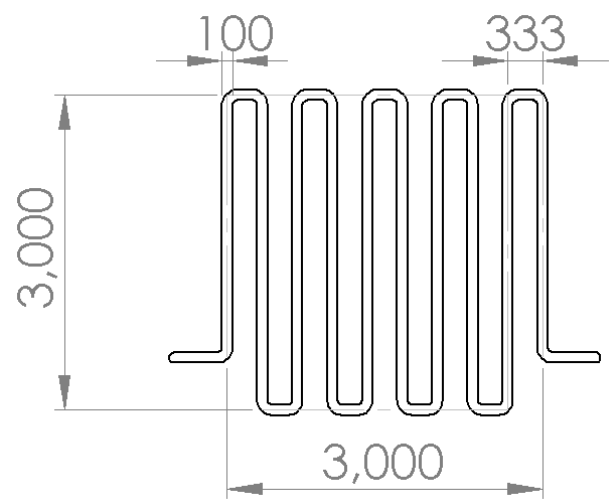

b)

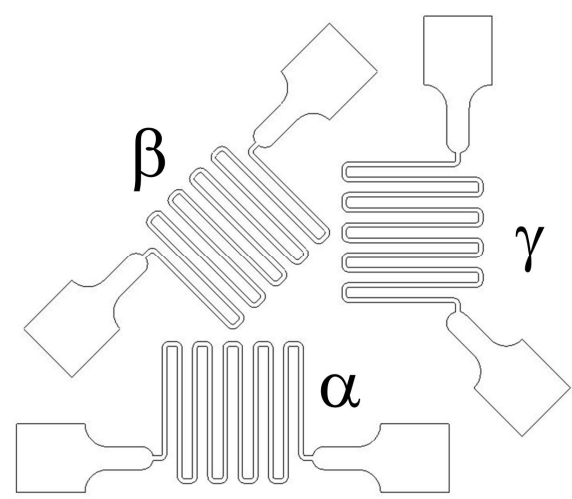

Figure 1. a) Geometry and dimensions of analyzed single sensor elements. Dimensions in $\mu \mathrm{m} . \mathrm{b}$ ) Configuration of sensor elements on each specimen.

To evaluate the impact of sensing element orientation relative to the substrate, experimental specimen featured three single elements arranged as in Fig. 1 b). Specimens for VSM measurements and thus the determination of $B$ - $H$-curves consisted of rectangular, $8 \times 3 \mathrm{~mm}$ large, permalloy thin films on corresponding substrates.

Structured permalloy thin films were fabricated by photolithography and lift-off processing. First, a photo mask was applied on the prepared substrates. Secondly, a permalloy thin film with a nominal thickness of $100 \mathrm{~nm}$ was deposited using sputtering (parameters: rf power: $200 \mathrm{~W}$, base pressure: $10^{-7}$ torr, Argon flow rate: 50 sccm). Consequently, photo mask and undesired permalloy coating were removed. After thin film processing, the AMR sensors were diced using a wafer saw, resulting in specimens comprising three individual sensor elements in the configuration depicted in Figure 1 b).

\section{B. Analytical Methods}

In order to characterize the polyimide-based substrates taken into consideration surface roughness was determined for each polyimide substrate material and a silicon wafer with a thermal oxide top layer, respectively. Surface roughness was measured as line roughness according to EN 25178 using a confocal laser scanning microscope (CLSM) (Keyence VK9700). Measurements were performed on substrates with a sputtered permalloy thin film on top. The metallic thin film was used due to its good reflection properties in order to improve the reliability of optical surface measurements and to avoid negative effects caused by the partial transparency of the used polymeric substrates. Furthermore, radius of curvature of the silicon carrier wafer was determined before and after application of the polymeric layers and the permalloy thin film by means of a tactile profilometer (Veeco Dektak ${ }^{3} \mathrm{ST}$ ). Using Stoney's equation, the difference between both measurements allowed an estimation of the magnitude and orientation of film stress within the polymeric layers. For spin-on precursors, a rather homogenous, axially symmetric state of stress was expected. Due to the method of application of the Kapton ${ }^{\circledR} \mathrm{HN}$ foil, a predominant direction of tensile stress has to be expected within the direction of application.

During magnetic characterization experiments, the samples were mounted on a fixture of a Helmholtz coil and a homogenous alternating magnetic field generated by a Helmholtz coil was applied either parallel, perpendicular or at $45^{\circ}$ to the specimen. The samples were operated in a Wheatstone bridge, with the output voltage amplified using an amplifier developed in-house. Changes of the electrical resistance as a function of the applied magnetic field were recorded simultaneously for each of the three sensor elements on a specimen using the logging function of a commercially available oscilloscope (Tektronix TDS 2014). Resulting $R$ - $H$-curves, which represent an alternating hysteresis, were evaluated in regard to the magnitude of the magnetoresistive effect ( $\Delta R / R_{0}$-ratio), as well as the shift of the $\Delta R / R_{0}$-ratio peaks relative to the applied field.

To determine effects of film stress on magnetic properties, hysteresis curves measurement were done on specimens using a vibrating sampling magnetometer (EG\&G Princeton Applied Research VSM Model 4500). Specimens of a size of 8 x $3 \mathrm{~mm}$ with $100 \mathrm{~nm}$ nominal thickness were mounted on a sample holder so that a measurement magnetic field was 
perpendicular to a film plan and parallel to the width of the specimens.

\section{RESULTS}

\section{A. Surface Roughness}

The surface roughness parameters arithmetical mean roughness $R_{a}$ and ten-point mean roughness $R_{z}$ of the analyzed substrates obtained by CLSM measurements are specified in Table 1.

Table 1. Surface roughness of analyzed substrates

\begin{tabular}{|c|c|c|}
\hline \multirow{2}{*}{ Substrate Material } & \multicolumn{2}{|c|}{ Surface Roughness } \\
\cline { 2 - 3 } & $\boldsymbol{R}_{\boldsymbol{a}}$ in $\boldsymbol{\mu m}$ & $\boldsymbol{R}_{\boldsymbol{z}}$ in $\boldsymbol{\mu m}$ \\
\hline $\mathrm{SiO}_{2}$ & 0.0088 & 0.0565 \\
\hline $\mathrm{Kapton}^{\circledR} \mathrm{HN}$ & 0.0675 & 0.1867 \\
\hline Spin-on precursor A & 0.0436 & 0.1310 \\
\hline Spin-on precursor B & 0.0982 & 0.3967 \\
\hline Spin-on precursor C & 0.0997 & 0.3042 \\
\hline
\end{tabular}

As expected, the values of surface roughness of the $\mathrm{SiO}_{2}$ substrate were significantly lower than those of the polyimide based materials. Best results for a polyimide based substrate were obtained for spin-on precursor A, followed by Kapton ${ }^{\circledR} \mathrm{HN}$ foil, spin-on precursor B and spin-on precursor C, respectively.

\section{B. Film Stress}

The determined film stress of the various polymeric substrates is depicted in Figure 2. Magnitude of stress is represented in relation to the estimated stress in the $\mathrm{SiO}_{2}$ specimen without polymeric coating. All samples show tensile stress, the Kapton ${ }^{\circledR} \mathrm{HN}$ sample shows an asymmetric behavior.

Estimated film stresses of spin-on precursor $\mathrm{A}$ and $\mathrm{C}$ significantly exceed those estimated for spin-on precursor $\mathrm{B}$. While the estimated film stress in spin-on precursor $\mathrm{B}$ only exceeds those estimated for $\mathrm{SiO}_{2}$ by 1.3 times, the estimated film stress in spin-on precursor A is 2.9 times higher and in spin-on precursor $\mathrm{C}$ it is even 5.8 times higher. An asymmetric deformation of the carrier wafers of Kapton ${ }^{\mathbb{B}} \mathrm{HN}$ foils could be observed by measuring carrier wafer deflection parallel and orthogonal to the direction of lamination resulting in a tensile stress in the direction of lamination half as high as in $\mathrm{SiO}_{2}$ and a compressive stress orthogonal to the direction of lamination double the stress value of $\mathrm{SiO}_{2}$. Thus, an inhomogeneous state of stress within the foils can be assumed. However the intermediate glue layer between silicon and Kapton ${ }^{\circledR} \mathrm{HN}$ foil likely had an influence on the warping as well by avoiding a full transformation of the foil's stress into the silicon wafer.

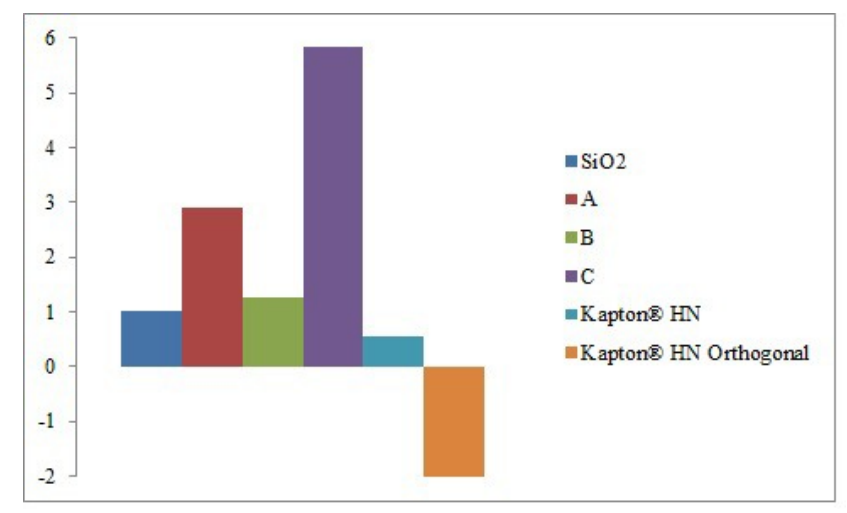

Figure 2. Estimated film stress in polymeric substrates in relation to non-coated $\mathrm{SiO}_{2}$ wafers. 


\section{Magnetization Curves}

Averaged values and standard errors of the characterstic parameters of $R$ - $H$-curves (base resistance, AMR effect ( $\Delta R /$ $R_{0}$-ratio) in \%, shift of $\Delta R / R_{0}$-ratio peaks along the $H$-field) are specified for all analyzed specimens in Table 2 . Table 3 shows characteristic $R$ - $H$-curves of sensor specimens of each of the analyzed substrates when an alternating $H$-field in the directions indicated in the first row had been applied.

As expected, best results were obtained on $\mathrm{SiO}_{2}$-substrates. In correlation to the lowest surface roughness determined for the $\mathrm{SiO}_{2}$ substrate, lowest base resistance was observed for these sensor elements as well (see Table 2). Furthermore, largest $\Delta R / R_{0}$-ratio and smallest shift of the $\Delta R / R_{0}$-ratio peaks along the $H$-field axis were determined. Thus, the magnetization curves of these $\mathrm{SiO}_{2}$ based sensor elements showed characteristics that are similar to those predicted by theory when fields along the hard and easy magnetization axes of such single sensor elements are applied. For example, considering the $\mathrm{SiO}_{2}$-specimen in $90^{\circ}$ orientation shown in Table 3: sensor element $\alpha$, oriented perpendicular to the $\mathrm{H}$ field, shows a typical transfer characteristic of an AMR permalloy thin film magnetoresistor [9]. Respectively, sensor element $\gamma$, oriented parallel to the $H$-field, shows characteristics of the planar Hall effect predicted by literature in this configuration. Respectively, the magnetization curves of these sensor elements switch when the $H$-field orientation is rotated about $90^{\circ}$. Sensor element $\beta$ shows an overlap of the planar or pseudo Hall effect with a lessened AMR effect in $0^{\circ}$ and $90^{\circ}$ orientation, a typical behavior of a magnetoresistor in an $H$-field oriented $45^{\circ}$ to its anisotropy axis. In the last configuration, when the H-field was applied perpendicular to the anisotropy axis of element $\beta$, the typical AMR effect transfer characteristics were measured as well.

Comparing sensor elements on polymeric substrates to the reference samples on $\mathrm{SiO}_{2}$ substrate, various changes of magnetization curves can be observed. First, the maximum $\Delta R / R_{0}$-ratios are reduced significantly. Second, an increase of field strengths at which the $\Delta R / R_{0}$-ratio maxima occur was identified. Third, the transfer characteristic of the sensor elements can no longer be purely attributed to shape anisotropy. While each appearance of an AMR effect maximum of the sensor elements $\alpha, \beta$ and $\gamma$ on $\mathrm{SiO}_{2}$ substrates can be distinctively related to a specific orientation of the $H$-field, this is no longer possible for sensor elements on polymeric substrates. Other factors besides shape anisotropy have to be taken into consideration. Thus, a more detailed look into the properties of the analyzed polymeric substrates is necessary. Although spin-on precursor A obtained best results concerning both surface roughness parameters $R_{a}$ and $R_{z}$ (see Table 1), sensor elements deposited on this substrate material exhibited the highest base resistance, performed worst in respect to $\Delta R / R_{0}$-ratio and shift of $\Delta R / R_{0}$-ratio maxima along the $H$-field (see Table 2) respectively. On the contrary, even though by far highest film stress (see Figure 2) and mediocre surface quality (see Table 1) were obtained for spin-on precursor $\mathrm{C}$, the average performance concerning base resistance and magnetic characteristics of the respective sensor elements was still better than those on spin-on precursor A. Worst surface quality was identified for spin-on precursor B. However, lowest film stress has to be expected following Stoney's equation (see Figure 2). In comparison to the other polymeric substrates, corresponding specimens performed clearly best in respect to base resistance and magnetic characteristics.

Nevertheless, the magnetization curves of all samples deposited on polymeric substrates showed asymmetric transfer characteristics. Thus, magnitudes and field strengths at which the $\Delta R / R_{0}$-ratio maxima occurred weren't identical for reversion from negative to positive $H$-fields and vice versa. According to literature, assymmetric transfer characteristics, or a dispersion of anisotropy [9] of this kind, are due to wall state transitions that occur in multidomain structures. In other words, when the applied magnetic field is reversed, Néel wall segments nucleate and expand which causes Barkhausen noise. Such Néel wall movement is known to be hindered by external factors such as surface roughness or film stress [15].

As mentioned above, another phenomenon that was exposed by the analysis of $R$ - $H$-curves was the apparent extinction of shape anisotropy of the sensor elements. Magnetization curves and corresponding AMR effect of samples on spin-on precursor $\mathrm{A}$ and $\mathrm{C}$ revealed very poor transfer characteristics so that a further analysis of interacting effects seems not expedient. However, Kapton ${ }^{\circledR}$ HN foil and spin-on precursor B seem to be promising candidates to investigate the various factors that affect magnetic thin films on polymeric substrates.

Considering transfer characteristics of a Kapton ${ }^{B} \mathrm{HN}$ based specimen as can be depicted from $R$ - $H$-curves in the $90^{\circ}$ orientation, sensor elements $\alpha$ and $\gamma$ feature very similar changes of resistance even though these two are tilted at an angle of $90^{\circ}$. At the same time, both elements are only weakly influenced by an $H$-field when oriented in $0^{\circ}$. Therefore, 
we assume that the original magnetization of these sensor elements is determined by an additional factor, such as a strong unidirectional stress. This stress is introduced when the Kapton ${ }^{\circledR} \mathrm{HN}$ foil is applied onto the wafer carrier by lamination. In the future either this stress can be used to bias sensing elements and to precisely set sensing elements or measures to avoid this stress will have to be taken.

In respect of specimens on spin-on precursor $\mathrm{B}$, another transfer characteristic is observed. In comparison to the reference samples on $\mathrm{SiO}_{2}$ substrate, the sensitivity of elements $\alpha$ and $\gamma$ to applied $H$-fields is inverted (see orientations $90^{\circ}$ and $0^{\circ}$ ). For the present, this effect can neither be explained by surface roughness nor by the presence of a unidirectional film stress. Relatively high values of surface roughness were in fact determined for this substrate material (see Table 1). Nevertheless, the relatively high $\Delta R / R_{0}$-ratio contradict a strong impact of surface roughness on these magnetization curves. Since film stress estimations using Stoney's equation didn't confirm the presence of a strong film stress, further factors have to be taken into consideration. An option could be a chemical interaction of the metallic thin film with the polymeric substrate [16], although this effect probably wouldn't cause the inversion of sensitivity.

Table 2. Averaged values and standard errors of characterstic parameters of $R$ - $H$-curves for the investigated substrates.

\begin{tabular}{|c|c|c|c|c|}
\hline Substrate & Base resistance in $\mathrm{k} \Omega$ & Resistivity in $\mu \Omega / \mathrm{cm}$ & AMR effect in \% & Peak shift in A / m \\
\hline $\mathrm{SiO}_{2}$ & $2.12 \pm 0.07$ & $64 \pm 0.02$ & $0.89 \pm 0.09$ & $241 \pm 16$ \\
\hline Kapton $^{(\mathbb{R})} \mathrm{HN}$ & $2.66 \pm 0.07$ & $81 \pm 0.02$ & $0.35 \pm 0.08$ & $6,263 \pm 1,105$ \\
\hline Spin-on precursor $\mathrm{A}$ & $9.65 \pm 0.54$ & $292(134) \pm 0.16$ & $0.06 \pm 0.01$ & $8,466 \pm 1,370$ \\
\hline Spin-on precursor B & $3.84 \pm 0.05$ & $116 \pm 0.02$ & $0.63 \pm 0.02$ & $580 \pm 64$ \\
\hline Spin-on precursor $\mathrm{C}$ & $8.90 \pm 0.70$ & $267(150) \pm 0.02$ & $0.13 \pm 0.05$ & $6,642 \pm 1,341$ \\
\hline
\end{tabular}


Table 3. Characteristic $R$ - $H$-curves of tested AMR sensor elements on different substrates.

Horizontal axis: $H$-Field in $\mathrm{A} / \mathrm{m}$, vertical axis: $\Delta R / R_{0}$ in $\%$.

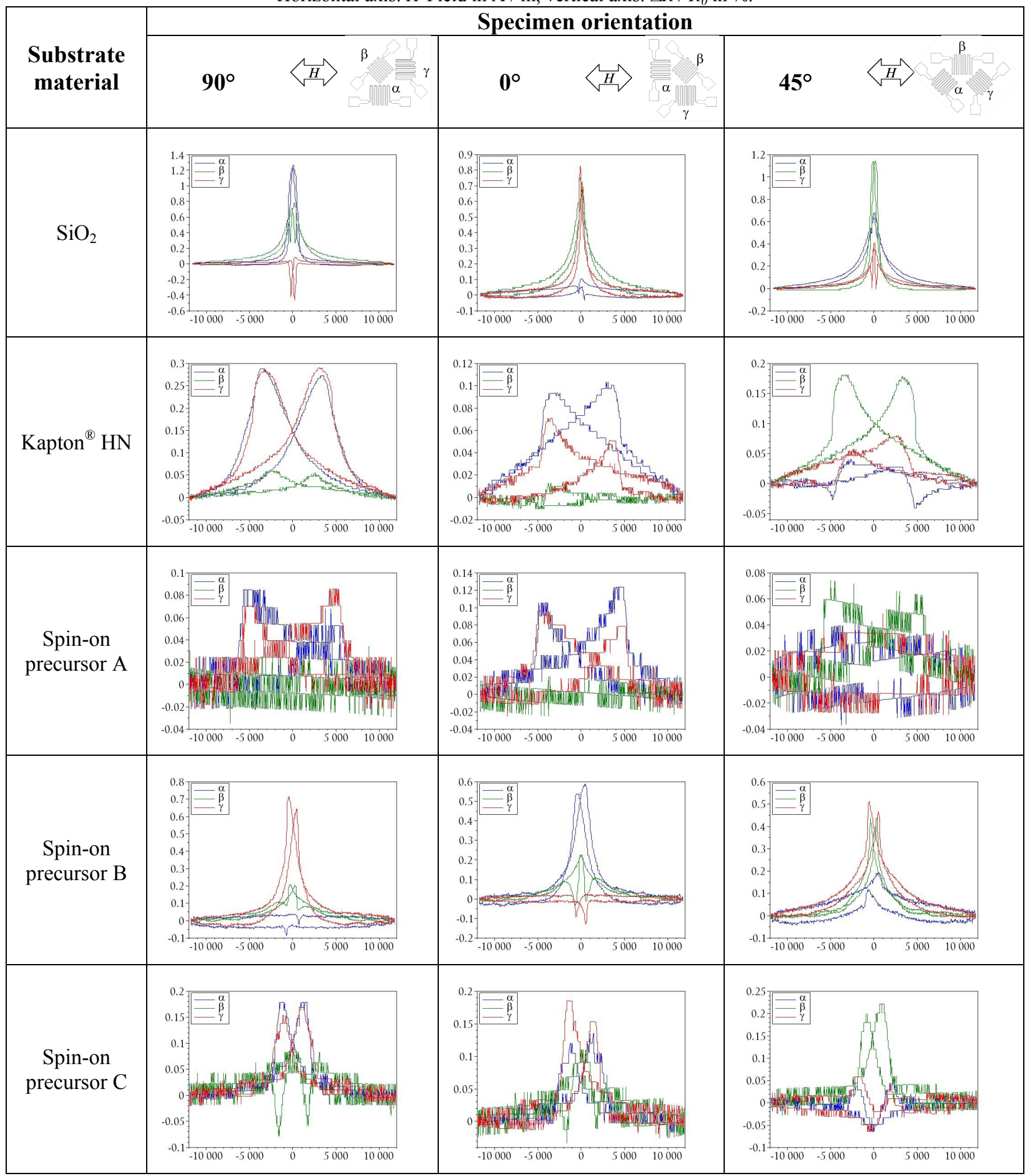




\section{VSM Measurements}

Results from VSM measurements (Figure 3) showed smaller coercivities $H_{c}$ of permalloy thin films deposited on $\mathrm{SiO}_{2}$, Kapton ${ }^{\circledR} \mathrm{HN}$ and spin-on precursor B compared to those deposited on spin-on precursor A and C as shown in Table 4. Two main factors originated from substrate which affect $H_{c}$ are surface roughness and film stress. The surface roughness causes domain wall pinning as described by Choe et al. in [3] for very thin permalloy film (thickness $<25 \mathrm{~nm}$ ). However for $100 \mathrm{~nm}$ thick permalloy films, the film stress has higher influence on $H_{c}$. Referring to Table 1 , all polymeric substrates possess high surface roughness but only permalloy films on Kapton ${ }^{\circledR} \mathrm{HN}$ and spin-on precursor B have small $H_{c}$. This is because their film stress is unidirectional thus defining overall magnetization direction of magnetic domains in the films. This also correlates to the $\Delta R / R_{0}$-ratio of Kapton ${ }^{\circledR} \mathrm{HN}$ in Table 1.

The saturation flux densities $B_{s}$ of the permalloy films deposited on $\mathrm{SiO}_{2}, \mathrm{Kapton}^{\circledR} \mathrm{HN}$ and spin-on precursor $\mathrm{B}$ are around $0.7 \mathrm{~T}$ while only $0.3-0.4 \mathrm{~T}$ are obtained from films deposited on spin-on precursor A and C. The reduced $B_{s}$ is a direct indicator of film thickness which is deviated from the $100 \mathrm{~nm}$ nominal value. The actual reduced film thickness is determined using ratio of $B_{s}$ of a substrate in question to $B_{s}$ of $\mathrm{SiO}_{2}$. Computed resistivities of specimens on $\mathrm{SiO}_{2}$ and Kapton $^{\circledR}$ HN listed in Table 2 coincide with those reported by Guittoum et al. [4]. Note also that the values in parentheses are computed using reduced film thickness.

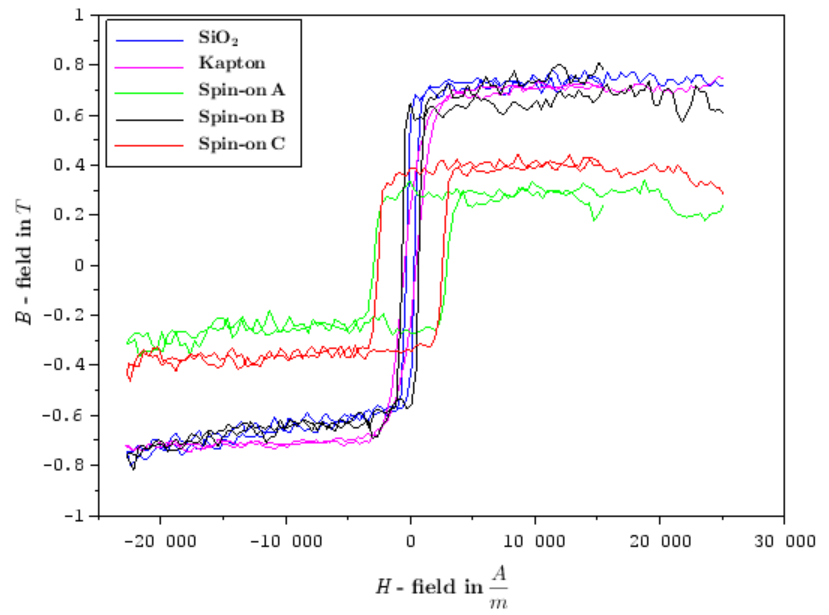

Figure 3. $B H$ hysteresis curves of specimens

Table 4. Coercivity values and saturation flux density of permalloy thin films on investigated substrates.

\begin{tabular}{|c|c|c|}
\hline Substrate & Coercivity $\boldsymbol{H}_{\boldsymbol{c}}$ in $\mathbf{A} / \mathbf{~ m}$ & Saturation Flux Density $\boldsymbol{B}_{\boldsymbol{s}}$ in T \\
\hline $\mathrm{SiO}_{2}$ & 317 & 0.72 \\
\hline Kapton $^{\circledR} \mathrm{HN}$ & 456 & 0.70 \\
\hline Spin-on precursor A & 2,940 & 0.33 \\
\hline Spin-on precursor B & 351 & 0.71 \\
\hline Spin-on precursor C & 2,584 & 0.40 \\
\hline
\end{tabular}




\section{CONCLUSION AND OUTLOOK}

We have investigated the impact of four different polyimide based substrates on the magnetic properties of permalloy thin film magnetoresistors by means of alternating $R$ - $H$-curves and $B$ - $H$-curves obtained by vibrating sample magnetometry. To further evaluate these substrates, surface roughness was determined using CLSM and film stress was estimated using Stoney's equation. Identical methods were applied to reference samples on $\mathrm{SiO}_{2}$ substrates.

$\operatorname{Kapton}^{\circledR} \mathrm{HN}$ and spin-on precursor B were exposed as promising substrate materials for permalloy thin film magnetoresistors. Although for $100 \mathrm{~nm}$ thin permalloy films, film stress was identified as more critical factor for a successful implementation of thin film magnetoresistors than surface roughness, future investigations will focus on a detailed qualification of stress state in spin-on precursor B.

\section{ACKNOWLEDGMENT}

This research has been sponsored in part by the German Research Foundation (DFG) within the Collaborative Research Center (SFB) 653 "Gentelligent Components in their Lifecycle".

\section{REFERENCES}

[1] Lachmayer, R., Mozgova, I., Sauthoff, B. and Gottwald, P., "Product Evolution and Optimization Based on Gentelligent Components and Product Life Cycle Data," Smart Product Engineering Lecture Notes in Production Engineering, 685-694 (2013).

[2] Griesbach, T., Wurz, M. C. and Rissing, L., "Development and Fabrication of Modular Micro Sensors on Flexible Polymer Foils," 1st Joint International Symposium on System-Integrated Intelligence (2012).

[3] Choe, G. and Steinback, M., "Surface Roughness Effects on Magnetoresistive and Magnetic Properties of NiFe Thin Films," Journal of Applied Physics 85, 5777 (1999).

[4] Guittoum, A., Bourzami, A., Layadi, A. and Schmerber, G., "Structural, Electrical and Magnetic Properties of Evaporated Permalloy Thin Films: Effect of Substrate and Thickness," European Physical Journal Applied Physics 58, 20301 (2012).

[5] Malozemoff, A. P., "Mechanisms of exchange anisotropy," Journal of Applied Physics 63, 3874-3879 (1988).

[6] Phuoc, N. N., Xu, F., Ma, Y. and Ong, C.K., "Permalloy-FeMn exchange-biased multilayers grown on flexible substrates for microwave applications," Journal of Magnetism and Magnetic Materials 321, 2685-2690 (2009).

[7] Carey, M. J. and Berkowitz, A. E., "CoONiO superlattices: Interlayer interactions and exchange anisotropy with Ni81Fe19 (invited)," Journal of Applied Physics 73, 6892 (1993).

[8] Néel, L., "L'anisotropie superficielle des substances ferromagnétiques," C. R. Acad. Sci. Paris 237, 1468-1470 (1953).

[9] Tumanski, S., [Thin film magnetoresistive sensors], IOP Publishing, 33-45 (2001).

[10] Hubert, A. and Schäfer, R.; [Magnetic Domains], Springer, 138-139 (1998).

[11] Sander, D., Enders, A. and Kirschner, J., "Stress and Magnetic Properties of Surface and Ultrathin Films," Journal of Magnetism and Magnetic Materials 200, 439-455 (1999).

[12] Grimes, C. A., "Sputter Deposition Of Magnetic Thin Films Onto Plastic: The Effect Of Undercoat And Spacerlayer Composition On The Magnetic Properties Of Multilayer Permalloy Thin Films," IEEE transactions on magnetics 31, No. 6, (1995).

[13] Fernández, E., Kurlyandskaya, G. V, García-Arribas, A., and Svalov, A. V., "Nanostructured giant magnetoimpedance multilayers deposited onto flexible substrates for low pressure sensing," Nanoscale Research Letters 7:230 (2012)

[14] Wright, W. W. and Hallden-Abberton, M., [Ullmann's Encyclopedia of Industrial Chemistry], Wiley-VCH Verlag GmbH \& Co. KGaA, Weinheim, 39-58 (2012).

[15] Cullity, B. D., and Graham, C. D., [Introduction to magnetic materials], John Wiley \& Sons (2011).

[16] Strunskus, T., Grunze, M., Kochendoerfer, G., Wöll, C., "Identification of Physical and Chemical Interaction Mechanisms for the Metals Gold, Silver, Copper, Palladium, Chromium, and Potassium with Polyimide Surfaces," Langmuir 12, 2712-2725 (1996). 